[0212-7199 (2005) 22: 4; pp 185-187] ANALES DE MEDICINA INTERNA Copyright (C) 2005 ARAN EDICIONES, S.L.

AN. MED. INTERNA (Madrid) Vol. 22, N. ${ }^{\circ} 4$, pp. 185-187, 2005

\title{
Sarcoma histiocítico: caso clínico y revisión de la literatura
}

\author{
P. KHOSRAVI SHAHI, A. DEL CASTILLO RUEDA ${ }^{1}$
}

Servicios de Oncología Médica y de ${ }^{I}$ Medicina Interna II. Hospital General Universitario Gregorio Marañón. Madrid

\author{
HISTIOCYTIC SARCOMA: A CASE REPORT AND REVIEW OF THE \\ LITERATURE
}

\section{RESUMEN}

El sarcoma histiocítico es una neoplasia rara, y de etiología desconocida, que se caracteriza por la proliferación maligna de células neoplásicas, que presentan rasgos morfológicos e inmunofenotípicos propios de los histiocitos tisulares. El curso clínico del sarcoma histiocítico es habitualmente rápidamente progresivo. Los síntomas y signos del sarcoma histiocítico incluye la presencia de síntoma sistémicos inespecíficos (fiebre, pérdida de peso), hepatoesplenomegalia, adenopatías, obstrucción intestinal, exantema cutáneo y pancitopenia.

Presentamos el caso clínico de una paciente de 75 años, con fiebre, pérdida de peso, anorexia, y astenia, acompañado de esplenomegalia y pancitopenia. El examen de médula ósea reveló el diagnóstico de sarcoma histiocítico.

PALABRAS CLAVE: Sarcoma histiocítico. Pancitopenia. Lisozima. Verdadero linfoma histiocítico. CD68.

\begin{abstract}
Histiocytic sarcoma is a rare neoplasm, and its aetiology is unknown. It is a malignant proliferation of neoplastic cells showing immunophenotypic and morphologic features similar to tissue histiocytes. The clinical course of histiocytic sarcoma is usually agressive. The signs and symptoms of histiocytic sarcoma are systemic symptoms (fever, weight loss), hepatosplenomegaly, adenopathies, intestinal obstruction, rash and pancytopenia.

We present the case of a 75 years old woman, with fever, wieght loss, anorexia, fatigue, splenomegaly and pancytopenia. Bone marrow examination showed the diagnosis of histiocytic sarcoma.
\end{abstract}

KEY WORDS: Histiocytic sarcoma. Pancytopenia. Lysozyme. True histiocytic lymphoma. CD68.

Khosravi Shahi P, Del Castillo Rueda A. Sarcoma histiocítico: caso clínico y revisión de la literatura. An Med Interna (Madrid) 2005; 22. 185-187.

\section{INTRODUCCIÓN}

El sarcoma histiocítico es una neoplasia de etiología desconocida, que se caracteriza por la proliferación de células neoplásicas que presentan rasgos morfológicos e inmunofenotípicos propios de los histiocitos tisulares $(1,2)$. El sarcoma histiocítico cursa con afectación diseminada de los órganos del sistema mononuclear fagocítico, tales como hígado, bazo, ganglios linfáticos, intestino delgado y piel.

\section{CASO APORTADO}

Anamnesis: Presentamos el caso de una mujer de 75 años, con antecedentes personales de diabetes mellitus tipo 2 insulindependiente, hipertensión arterial, dislipemia, cardiopatía isquémica, apen- dicectomía y colecistectomía, que presentaba fiebre de 1 mes de evolución , acompañado en los últimos 6 meses de síndrome constitucional (anorexia, astenia y pérdida de unos 21 kilogramos de peso) y aparición de múltiples hematomas cutáneos ante mínimos traumatismos.

Exploración física: En la exploración física destacaba una intensa palidez mucocutánea, múltiples hematomas cutáneos, murmullo vesicular disminuido de manera generalizada con crepitantes en base pulmonar derecha en la auscultación pulmonar y esplenomegalia a la palpación, sin hepatomegalia, ni adenopatía periféricas.

Pruebas complementarias: Las pruebas complementarias aportaban los siguientes resultados:

-Hemograma: a) serie roja: hemoglobina $7,2 \mathrm{~g} / \mathrm{dl}$, hematocrito $22 \%$, hematíes 2.710.000/ul, VCM 81,1 fl, HCM 26,6 pg, CHCM $32,8 \mathrm{~g} / \mathrm{dl}$, amplitud de distribución eritrocitaria $19,8 \%$, reticulocitos $3,45 \%(107.000 / \mu \mathrm{l})$; b) serie plaquetaria: plaquetas $11.000 / \mu \mathrm{l}$; y c)serie blanca: leucocitos $1.500 / \mu l$. 
-Frotis de sangre periférica: a) serie roja: anisocitosis, $14 \%$ normoblastos, dacriocitos y algunos macroovalocitos; b) serie plaquetaria: plaquetas gigantes; c) serie blanca: $52 \%$ segmentados, $30 \%$ linfocitos, $4 \%$ monocitos.

-VSG: $32 \mathrm{~mm} /$ hora

-Bioquímica: glucosa $67 \mathrm{mg} / \mathrm{dl}$, urea $33 \mathrm{mg} / \mathrm{dl}$, creatinina 0,4 $\mathrm{mg} / \mathrm{dl}$, urato $5,6 \mathrm{mg} / \mathrm{dl}$, bilirrubina $1,1 \mathrm{mg} / \mathrm{dl}$, AST $141 \mathrm{UI} / \mathrm{l}$, ALT 38 UI/l, Fosfatasa alcalina 245 UI/l, LDH 1650 UI/L, GGT 75 UI/l, proteínas totales $4,9 \mathrm{mg} / \mathrm{dl}$, colesterol $149 \mathrm{mg} / \mathrm{dl}$, triglicéridos 266 $\mathrm{mg} / \mathrm{dl}$, calcio $9,1 \mathrm{mg} / \mathrm{dl}$, hierro $62 \mu \mathrm{g} / \mathrm{dl}$, indice de saturación de tranferrina $34 \%$, transferrina $143 \mathrm{mg} / \mathrm{dl}$, ferritina $7597 \mu \mathrm{g} / \mathrm{dl}$, vitamina B12 $609 \mathrm{pg} / \mathrm{ml}$, folato $1,81 \mathrm{ng} / \mathrm{ml}$, alfafetoproteína $<1$.

-Proteinograma: albúmina $54,6 \%$, alfa 1 globulina $3 \%$, alfa 2 globulina $11,8 \%$, beta globulina $15,4 \%$, gamma globulina $15,2 \%$.

-Radiografía de torax: infiltrado pulmonar basal derecho.

-Ecografía abdominal: múltiples lesiones focales hepáticas con afectación difusa del parénquima hepático con tamaño hepático normal, marcada esplenomegalia, sin que se visualizen adenopatías intrabdominales.

Diagnóstico: Así pues, ante la presencia de un cuadro de síndrome constitucional, fiebre, pancitopenia con datos de infiltración de médula ósea, mieloptisis (reacción leucoeritroblástica y dacriocitos en frotis de sangre periférica), y la presencia de lesiones ocupantes de espacio hepáticas se decidió realizar un aspirado y biopsia de médula ósea.

Sin embargo, antes de obtener el resultado definitivo del examen de médula ósea, la paciente a pesar del soporte hemoterápico, evolucionó de manera desfavorable falleciendo a los 9 días de su ingreso. No se llegó a realizar la autopsia por deseos expresos de la familia.

El examen de médula ósea evidenció los siguientes datos: brosis).

-Aspirado de médula ósea: aspirado seco (sugerente de mielofi-

-Biopsia de médula ósea: parénquima medular hipercelular (5/5), a expensas de una infiltración masiva y difusa de células neoplásicas de aspecto hematopoyético, con fenómenos de mielofibrosis y necrosis. Las células tumorales son de talla grande, citoplasma abundante eosinófilo y núcleos voluminosos y polimórficos, con nucleolo prominente central y abundantes figuras de mitosis. No se aprecian fenómenos de hemofagocitosis.

Inmunohistoquímica:

- CD45/ EMA/ LISOZIMA (patrón Golgi)/ CD68: positivos.

- CD79a/ CD10/ CD23/ CD43/ IgS/ CD15/ CD30/ CD45RO/ CD1a/ CD2 / CD3/ CD4/ CD8/ CD57/ CD 34/ MPX/ FACTOR VIII/ LMP1 (VEB)/ BCL2/ P53: negativos.

- Tasa de proliferación celular $(\mathrm{Ki} 67)>80 \%$.

Las células tumorales se acompañan de células reactivas linfoides de estirpe $\mathrm{T}$ (CD45RO/ CD2/CD3/CD8/CD57 positivos) e histiocitos (CD68 positivo).

El diagnóstico definitivo del examen de médula ósea era compatible con un sarcoma histiocítico según la clasificación de la Organización Mundial de la Salud.

\section{DISCUSIÓN}

La presencia de fiebre, síndrome constitucional, pancitopenia y esplenomegalia en una paciente anciana debe incluir en su diagnóstico diferencial las enfermedades que se reflejan en la tabla I.

El sarcoma histiocítico se puede dar a cualquier edad, pero la mayoría de los casos tienen lugar en adultos, con una mediana de edad de 45 años. No hay un predominio sexual claro. El curso clínico del sarcoma histiocítico es rápidamente progresivo, pudiendo ser fulminante en ocasiones, como lo fue en nuestro caso.

\begin{tabular}{l} 
TABLA I \\
DIAGNÓSTICO DIFERENCIAL DE PANCITOPENIA Y \\
ESPLENOMEGALIA \\
\hline 1. Tricoleucemia o leucemia de células peludas \\
2. Linfoma no Hodgkin de zona marginal esplénica \\
3. Leucemia prolinfocítica \\
4. Enfermedades infecciosas: tuberculosis diseminada, \\
leishmaniosis visceral o Kala-Azar, brucelosis, etc. \\
5. Amiloidosis \\
6. Sarcoidosis \\
7. Neoplasias sólidas con metástasis a distancia e infiltra- \\
ción de médula ósea (mama, pulmón, colon, etc.) \\
8. Otros: malaria crónica, síndrome de Felty, neoplasias \\
de células dentríticas e histiocitarias (sarcoma histiocí- \\
tico), etc.
\end{tabular}

Los pacientes pueden presentar síntomas sistémicos inespecíficos como fiebre, mal estado general, anorexia, astenia y pérdida de peso (síntomas presente en nuestro caso). Otros datos comunes son la hepatoesplenomegalia (3) (esplenomegalia marcada en nuestro caso), adenopatías y lesiones cutáneas (4).

Puede haber afectación de diversos órganos, tales como hígado y bazo (hepatoesplenomegalia, alteración de las enzimas hepáticas), médula ósea (mieloptisis y citopenias hematológicas, presente en nuestro caso), intestino delgado (obstrucción intestinal, malabsorción, diarrea crónica). Aunque son más raros, puede afectar también a los huesos (lesiones osteolíticas, dolor óseo), infiltrados pulmonares y/o afectación del sistema nervioso central (5).

Para establecer el diagnóstico definitivo es preciso realizar un estudio anatomopatológico de uno de los órganos afectados: biopsia de médula ósea si datos de mieloptisis (como en nuestro caso); de ganglios linfáticos si adenopatías; intestino delgado si diarrea u masa intestinal o de la piel; con determinación inmunofenotípica e incluso estudios genéticos.

El examen anatomopatológico suele revelar la desestructuración de la arquitectura normal del órgano, debido a una infiltración difusa de células neoplásicas de gran tamaño, polimórficas, de contorno redondo u ovalado, y de citoplasma amplio y eosinófilo, con núcleos grandes y excéntricos. Con frecuencia se observan células gigantes multinucleadas. El aspecto microscópico global puede ser indistinguible de los linfomas de alto grado (6). Los fenómenos de hemofagocitosis son escasos y suele existir un número variable de células reactivas acompañando a las células reactivas.

Para establecer el diagnóstico definitivo es necesario el estudio inmunofenotípico, y por definición debe existir expresión de uno o más marcadores histiocitarios (CD68, alfa1antitripsina, lisozima, CD11c, CD14), con ausencia de marcadores de células dentríticas/ Langerhans (CD1a, CD21, CD35), de los linfocitos B y T (CD2, CD3, CD8, CD19, CD20, CD22, CD79a), y de los marcadores mieloides específicos (mieloperoxidasas, CD13, CD33,CD34) $(2,7,8)$. La lisozima suele presentar un patrón granular intracitoplasmático con una mayor acentuación habitualmente a nivel de aparato de Golgi (patrón Golgi positivo). 
Los estudios genéticos de reordenamiento de los genes de las cadenas pesadas de las inmunoglobinas (PCR de $\operatorname{IgH}$ ) y del gen de la cadena gamma del receptor de los linfocitos $\mathrm{T}$ (TCR) son negativos (9).

En la mayoría de los casos el tumor se presenta en un estadio avanzado (III-IV), como es nuestro caso. En los estadios avanzados es preciso recurrir a la poliquimioterapia, siendo el esquema más empleado CHOP. Algunos autores añaden otros agentes como bleomicina, etopósido o ara- binósido de citosina. Más reciente se ha descrito la utilidad de talidomida en las neoplasias hematológicas $(10,11)$. Dalle y cols. han utilizado la talidomida en un sarcoma histiocítico en un niño, obteniendo una buena respuesta clínica (12).

Como conclusión hay que mencionar que el sarcoma histiocítico a pesar de ser un proceso infrecuente debe ser tenida en cuenta si hay pancitopenia, fiebre sin focalidad, esplenomegalia y /o adenopatías.

\section{Bibliografía}

1. Elghetany MT. True histiocytic lymphoma: is it an entity? Leukemia. 1997; 11: 762-4.

2. Copie-Bergman C, Wotherspoon AC, Norton AJ, Diss TC, Isaacson PG. True histiocytic lymphoma: a morphologic, immunohistochemical, and molecular genetic study of 13 cases. Am J Surg Pathol 1998; 22: 1386-92.

3. Kimura H, Nasu K, Sakai C, Shiga Y, Miyamoto E, Shintaku M, et al. Histiocytic sarcoma of the spleen associated with hypoalbuminemia, hypo gamma-globulinemia and thrombocytopenia as a possibly unique clinical entity-report of three cases. Leuk Lymphoma 1998; 31: 217 24.

4. Osborne BM, Mackay B. True histiocytic lymphoma with multiple skin nodules. Ultrastruct Pathol 1994; 18: 241-6.

5. Sun W, Nordberg ML, Fowler MR. Histiocytic sarcoma involving the central nervous system: clinical, immunohistochemical, and molecular genetic studies of a case with review of the literature. Am J Surg Pathol 2003; 27: 258-65.

6. Pileri S, Mazza P, Rivano MT et al. Malignant histiocytosis (true histiocytic lymphoma) clinicopathological study of 25 cases. Histopathology 1985; 9: 905-20.
7. Pileri SA, Grogan TM, Harris NL, Banks P, Campo E, Chan JK,et al. Tumours of histiocytes and accessory dendritic cells: an immunohistochemical approach to classification from the International Lymphoma Study Group based on 61 cases. Histopathology 2002; 41: 1-29.

8. Krenacs L, Tiszalviez L, Krenacs T, Boumsell L. Immunohistochemical detection of CD1a antigen in formalin-fixed and paraffin-embedded tissue sections with monoclonal antibody 010. J Pathol 1993; 100: 285-92.

9. Ralfkiaer E, Delsol G, O'Connor NTJ, Brandtzaeg P, Brousset P, Vejlsgaard Gl, et al. Malignant lymphomas of true histiocytic origin. A clinical, histological, immunophenotypic and genotypic study. J Pathol 1990; 160:9-17.

10. Rajkumar SV, Dispenzieri A, Fonseca R, Lacy MQ, Geyer S, Lust JA et al. Thalidomide for previously untreated indolent or smoldering multiple myeloma. Leukemia 2001; 15: 1274-1276.

11. Baidas S, Tfayli A, Bhargava P. Thalidomide: an old drug with new clinical applications. Cancer Invest 2002; 20: 835-848.

12. Dalle JH, Leblond P, Decouvelaere A, Yakoub-Agha I, Preudhomme C, Nelken B, et al. Efficacy of thalidomide in a child with histiocytic sarcoma following allogeneic bone marrow transplantation for T-ALL. Leukemia 2003; 17: 2056-7. 